\title{
Interval-valued Fuzzy Sets in Soft Computing
}

\author{
Humberto Bustince \\ Depatamento de Automática y Computación, \\ Universidad Pública de Navarra, \\ Campus Arrosadia, $s / n$, \\ Pamplona, Navarra 31006, Spain \\ E-mail: bustince@unavarra.es \\ Received: 06-11-2009 \\ Accepted: 28-05-2010
}

\begin{abstract}
In this work, we explain the reasons for which, for some specific problems, interval-valued fuzzy sets must be considered a basic component of Soft Computing.
\end{abstract}

Keywords: Fuzzy set; Interval-valued fuzzy set; Type-2 fuzzy set; Soft Computing.

\section{Introduction}

The question: What is Soft Computing? has been answered by Magdalena in FLINS'08 in the work: What is Soft Computing? Revisiting Possible Answers $\left(\right.$ see $\left.^{1}\right)$.

From the analysis of the different reflexions about the defining limits of Soft Computing (SC) in $2,3,1,4,5,6,7$, we note that each time there is an effort to extend the number of components of SC. Taking into account this fact, the aim of this work is to show that there exist some situations where intervalvalued fuzzy sets (IVFSs), also called interval type2 fuzzy sets, must be considered as one of the basic components of SC.

In Engineering, we use different levels of models to solve a problem, depending on its complexity. In our case, the choice of level (with respect to the basic components of SC) depends on the uncertainty associated to the problem we are considering. Moreover, the complexity of the selected model must be a function, on one hand, of the degree of accuracy we want to get for the solution; and on the other hand, of the degree of knowledge at our disposal to build the membership functions of the elements of the fuzzy sets (FSs) ${ }^{8}$, also called type- 1 fuzzy sets.

The use of IVFSs or FSs should depend on the problem under consideration and the model level used to solve it. That is, we consider that it is not appropriate to impose the use of one or another type of sets for every problem. In those cases where the solution is almost the same using any of these two types of sets it is advisable to use FSs, since they are simpler to handle and they have been much more studied.

\section{In soft computing the writing instrument must be a spray pen}

In November, 24, 2008 Zadeh proposed in the Berkeley Initiative in Soft Computing (bisc-group list), the following definition of SC:

Soft computing is a coalition of methodologies which collectively constitute a system with wideranging capabilities for the conception, design and 
operation of intelligent systems. The principal members of the coalition are: fuzzy logic, neurocomputing, evolutionary computing and probabilistic computing. The guiding principle of soft computing is: In general, better results can be achieved through the use of constituent methodologies of soft computing in combination rather than isolation.

From the analysis of this definition and of the work of ${ }^{1}$ we deduce three conclusions:

i) The concept of $\mathrm{SC}$ is not a static one, it evolves along time.

ii) SC is always defined by extension, using different theories that have in common that all of them are used to solve problems that take into account imprecision in the world around us and subjectivity associated to human being.

iii) Fuzzy Logic is always a component of SC.

Regarding fuzzy logic, Zadeh suggests the following definition: (December, 11, 2008, bisc-group list)

Fuzzy logic is a precise system of reasoning, deduction and computation in which the objects of discourse and analysis are associated with information which is, or is allowed to be, imperfect.

Imperfect information is defined as information which in one or more respects is imprecise, uncertain, vague, incomplete, partially true or partially possible.

In the same date, Zadeh made the following remarks

1. In fuzzy logic everything is or is allowed to be a matter of degree. Degrees are allowed to be fuzzy.

2. Fuzzy logic is not a replacement for bivalent logic or bivalent-logic- based probability theory. Fuzzy logic adds to bivalent logic and bivalent-logic-based probability theory a wide range of concepts and techniques for dealing with imperfect information.

3. Fuzzy logic is designed to address problems in reasoning, deduction and computation with imperfect information which are beyond the reach of traditional methods based on bivalent logic and bivalent-logic - based probability theory.

4. In fuzzy logic the writing instrument is a spray pen with precisely known adjustable spray pattern. In bivalent logic the writing instrument is a ballpoint pen.

5. The importance of fuzzy logic derives from the fact that in much of the real world imperfect information is the norm rather than exception.

In the world of Logic, we know that given a predicate $P$ on a universe $U=\{x, y, z, \cdots\}$, there exists a compatibility degree between $P$ and objects in $U$ which provides the truth degree of proposition " $x$ is $P$ ".

If we accept the existence of propositions (" $x$ is $P$ ") that true or false in some degree, we must extend our logic to the existence of another subsets apart from the classical ones $T$ (True) and $F$ (False). That is, we must think of multivalued logics and not only of bivaluate ones. In case we deal with imperfect information, we must use as basic logic the fuzzy logic. Having in mind these considerations, the following is settled in ${ }^{9}$ :

Given a predicate $P$ in a universe of discourse $U$, we suppose that there exists a mapping $\mu_{\widetilde{P}}: U \rightarrow[0,1]$ such that $\mu_{\widetilde{P}}(x)$ properly represents the value of the proposition " $x$ is $P$ " for all $x \in U$. Under these conditions, we will speak of the Fuzzy Set $\widetilde{P}$ defined by the mapping $\mu_{\widetilde{P}}$; that is,

$$
\widetilde{P}=\left\{\left(x, \mu_{\widetilde{P}}(x)\right) \mid x \in U\right\}
$$

Once this definition of FS has been accepted, from a mathematical point of view we can develop a fuzzy mathematical theory based on this concept. In such a theory, it is accepted that we know the membership degree of the elements to the set. However, in the applied world, it often happens that the membership function $\mu_{\widetilde{p}}$ is imperfect information and it is not known in an accurate way. For instance, in image processing $\left(\right.$ see $^{10}$ ) the success of the application of FSs depends on the knowledge that an expert 
has to build the degrees of membership of pixels to the FS representing the image.

These considerations, together with notes (1)-(5) of Zadeh, lead us to say that, in applications where the expert does not have a precise knowledge of the membership function to use for building the FSs, it is appropriate to represent the membership degree of each element to the fuzzy set by means of an interval. From these considerations arises the extension of fuzzy sets called theory of interval-valued fuzzy sets, that is, fuzzy sets such that the membership degree of each element of the fuzzy set is given by a closed subinterval of the interval $[0,1]$. Hence, not only vagueness (lack of sharp class boundaries), but also a feature of uncertainty (lack of information) can be addressed intuitively (see ${ }^{11}$ ).

We know that most enumerations of SC components include Fuzzy, Neural, Probabilistic and Evolutionary Computation as the four basic components. Nevertheless, if in a particular application it is very important to build the membership function with "very good" accuracy, and if the experts do not know how to do it, then we must use IVFSs. From these reflections and from the atudy done in ${ }^{12}$ we propose the graphics in Fig. 1. Analyzing that figure, we deduce that we can also build hybrid approaches using IVFSs, as, for instance, fuzzy neural networks using IVFSs ( see $^{13}$ )

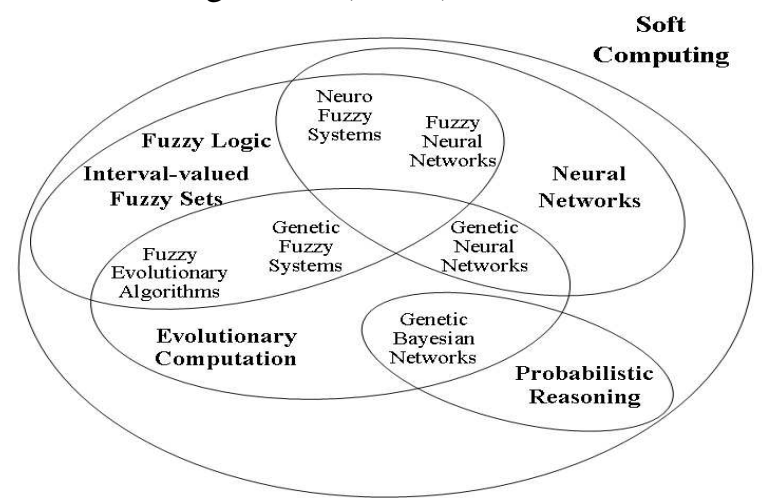

Fig. 1. A graphical view of Hybridization ( see $^{2}$ )

\section{A Note about Interval-valued fuzzy sets}

These sets were born in the 1970s. In May 1975 Sambuc $\left(\right.$ see $^{14}$ ) presented in his doctoral thesis the concept of an interval-valued fuzzy set named a $\Phi$ - fuzzy set. That same year, Jahn ${ }^{15}$ wrote about these sets and Zadeh ${ }^{16}$ discussed the representation of type-2 fuzzy sets (fuzzy sets such that the membership of each element to the set is given by another fuzzy set) and its potential in approximate reasoning. One year later, Grattan-Guinness ${ }^{17}$ established a definition of an interval-valued membership function. In that decade interval-valued fuzzy sets appeared in the literature in various guises and it was not until the 1980s, with the work of Gorzalczany and Türksen ${ }^{18}{ }^{28}$, that the importance of these sets, as well as their name, was definitely established.

Let us denote by $L([0,1])$ the set of all closed subintervals in $[0,1]$, that is,

$$
L([0,1])=\left\{\mathbf{x}=[\underline{x}, \bar{x}] \mid(\underline{x}, \bar{x}) \in[0,1]^{2} \text { and } \underset{-}{x} \leqslant \bar{x}\right\} .
$$

Definition 1. An interval-valued fuzzy set (IVFS) (or interval type-2 fuzzy set) $A$ on the universe $U \neq \emptyset$ is a mapping $A: U \rightarrow L([0,1])$, such that the membership degree of $u \in U$ is given by $A(u)=$ $[A(u), \bar{A}(u)] \in L([0,1])$, where $A: U \rightarrow[0,1]$ and $\bar{A}: U \rightarrow[0,1]$ are mappings defining the lower and the upper bound of the membership interval $A(u)$, respectively.

Basically, there exist two different interpretations of IVFSs:

1.- As mathematical entities such that the membership of each element to the set is given by a closed interval. Obviously, with this interpretation, we are working with two functions $A$ and $\bar{A}$, so we duplicate the complexity with respect to working with FSs. We consider this interpretation of interest only from a theoretical point of view.

2.- Each interval represents that the expert has a clear idea of a lower and an upper bound for the membership degree of an element to the fuzzy set, although he or she does not know which is the exact value of that membership. From our point of view, this interpretation is the one we must use when working with imprecise information about the degrees of membership to the FS. Therefore (see 
${ }^{29}$ ), for us, membership functions of intervalvalued fuzzy sets are not as specific as their counter-parts of fuzzy sets, but this lack of specificity makes them more realistic in some applications. Their advantage is that they allow us to express our uncertainty in identifying a particular membership function. This uncertainty is involved when interval-valued fuzzy sets are processed, making results of the processing less specific but more credible.

We think that the main problems of IVFSs nowadays are the following $\left(\right.$ see $\left.^{11}\right)$ :

- A large number of contributions are generalized adaptations of the theoretical developments of fuzzy set theory. This prevents us from focusing on the nature of interval-valued fuzzy sets themselves and studying the properties possessed exclusively by these sets.

- In the early work on this theory, Gorzalczany presented the concept of the degree of compatibility between two interval-valued fuzzy sets as an interval (see ${ }^{19}$ ). In some of the latest publications that we have read on intervalvalued measures of information, these measures are defined as a point on $[0,1]$. With this modelization, what is achieved is that the relation between different interval-valued measures of information is a copy of the relation that exists between these concepts in fuzzy set theory. However, we consider that in this case we lose the information that would be provided by a modelization of these measures using intervals.

- Currently, there are two names for these sets, some authors call them interval-valued fuzzy sets and others interval type-2 fuzzy sets. In ${ }^{30}$ Mendel writes: It turns out that an interval type-2 fuzzy set is the same as an intervalvalued fuzzy set for which there is a very extensive literature. These two seemingly different kinds of fuzzy sets were historically approached from very different starting points, which as we shall explain next has turned out to be a very good thing. Nonetheless, we consider that this duplicity in the name can cause confusion. We have observed that in some papers results that have already been known for many years for interval-valued fuzzy sets are presented for interval type- 2 fuzzy sets. For this reason, we believe the name should be set, otherwise, the complete bibliography that exists on interval-valued fuzzy sets should be taken into account.

Other objections to these sets can be found in ${ }^{31}$.

However, taking into account that in present day the power of computers is very big, we consider that it is advisable to use IVFSs instead of FSs in those problems where there exists a large imprecision in the information that we need the functions of membership to the FS.

\section{Applications of Interval-valued fuzzy sets}

In last years, many research works making use of IVFSs have been published. In this section we do not intend to make a precise description of all of them, but just to show the wide range of applications that thse works cover. We think that the importance of these applications provides another argument supporting inclusion of IVFSs as a basic component of SC. A feature of all the works we are citing is that results obtained when using IVFSs are better that those obtained when using FSs.

Approximate reasoning: ${ }^{32,33,19}$. Expert systems: ${ }^{34}$. Classification systems based on rules : 35,36 . Control: ${ }^{20,37} \_{ }^{41}$. Image processing: ${ }^{42,43}$. Redes neuronales: ${ }^{13}$. Intelligent web: ${ }^{44}$. Decision Making: ${ }^{45,46}$. Medicine: ${ }^{47,48}$. Mobile networks: ${ }^{49}$. Pattern recognition: ${ }^{50}$. Genetic algorithms: ${ }^{51}$.

\subsection{Compute with words and perceptions, Interval-valued fuzzy sets and Soft Computing}

Zadeh (see ${ }^{52}$ ) proposed the paradigm of computing with words (CWW) as a methodology in which the objects of computation are words and propositions draw from a natural language. Usually, words in the CWW paradigm have been modeled using FSs or its 
extension, type-2 fuzzy sets. We know that IVFSs are a particular type of type-2 fuzzy sets, and also that they are the most widely used amongst type- 2 fuzzy sets ( see $^{53}{ }^{55,30}$ ).

CWW using fuzzy sets has been studied by many researchers, however, the limitations of using fuzzy sets in CWW have also been pointed out by several researchers $\left(\right.$ see $^{56,57}$ ):

- Herrera and Herrera-Viedma (see ${ }^{12}$ ) state: Formally speaking, it seems difficult to accept that all individuals should agree on the same membership function associated to linguistic terms.

- Türksen (see ${ }^{58}$ ) points out: Fuzzy sets representation is a "reductionistic" approach for it discards the spread of membership values by averaging or curve fitting techniques and hence, camouflages the "uncertainty" embedded in the spread of membership values. Therefore, fuzzy sets representation does not provides a good approximation to meaning representation of word and does not allow computing with words a richer platform.

- Mendel (see ${ }^{59,60}$ ) notes: words mean different things to different people and so are uncertain. We therefor need a fuzzy set model for a word that has the potential to capture its uncertainties, and interval-valued fuzzy sets should be used as a fuzzy set model of a word. It also settles that it is scientifically incorrect to model words in the Perceptual Computer by the proposed one using fuzzy sets.

All these reasons, together with the applications mentioned above and with the fact that in ${ }^{1}$ (page 8) Magdalena points out that that CWW and Perceptions should be considered as a basic element of SC, support our statement that there exist some problems for which better solutions are achieved when IVFSs are considered a basic element of SC.

\section{Soft computing and other extensions}

We know that, apart from IVFSs, there exist other extensions of FSs (see ${ }^{61,62}$ ). In Figure 2 we show some of them. From a mathematical point of view, it has been proved that most of such extensions are equivalent to each other; for instance, Atanassov's intuitionistic fuzzy sets and IVFSs. However, from a conceptual point of view, each extension is different from the others, and each of them has originated to solve a different problem.

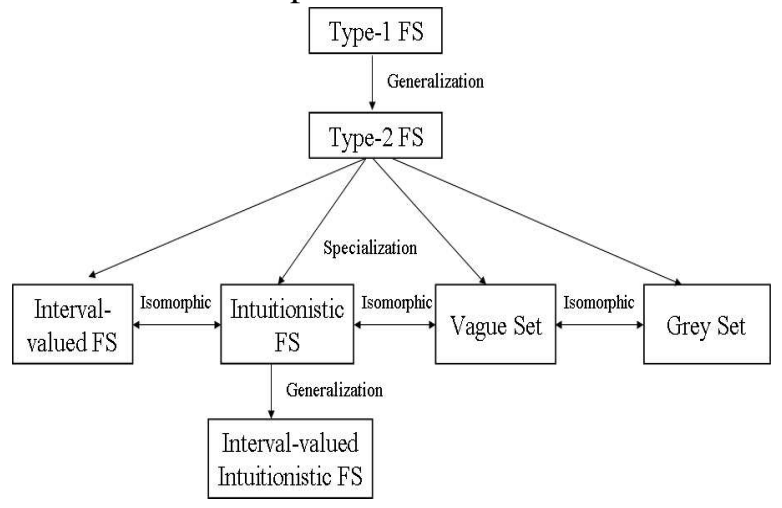

Fig. 2. Some extensions of FSs

The fact that in recent years IVFSs have been more applied and studied than the other extensions has led us to write this paper from the point of view of IVFSs. Nevertheless, we have already said that one of the main features of the SC concept is its evolutive character along time. We believe that in a nearby future, type- 2 fuzzy sets will be more studied, and, when the computer power becomes greater, they will be also the most widely used extensions of fuzzy sets. So we think that that in a few years type-2 fuzzy sets will have to be considered a basic constituent of SC. But today it is not even possible to suggest such possibility.

\section{Conclusions}

From all the comments we have made along this work, we conclude that:

a) When the accuracy in the construction of the membership function of the elements of the FS is not very determinant in the final result of the problem, we must use FSs, since they are easier to handle and they are much better studied.

b) If we use SC techniques to solve problems where we need a relatively precise solution and very conditrioned by the membership function we use, then 
we must impose that IVFSs are basic elements of SC.

Finally, we must remark that IVFSs are useful under appropriate conditions. We do not want to state that using them is always the best option. Once again, as Zadeh itself pointed out, we are in the presence of the famous hammer principle: when the only tool you have is a hammer, everything you find is a nail

\section{Acknowledgments}

This research was partially supported by the Grant TIN2007-65981.

\section{References}

1. L. Magdalena, "What is Soft Computing? Revisiting possible answers", Proceedings of the 8th International FLINS Conference, FLINS'08, World Scientific, 3-11 (2008).

2. O. Cordón, F. Gomide, F. Herrera, F. Hoffmann, L. Magdalena, "Ten Years of Genetic Fuzzy Systems: Current Framework and New Trends", Fuzzy Sets and Systems, 141 (1), 5-3 (2001).

3. Li, X., Ruan, D. and van der Wal, A.J., "Discussion on soft computing at FLINS'96", International Journal of Intelligent Systems, 13, 2-3, 287-300 (1998).

4. J.L. Verdegay,, Una revisión de las metodologias que integran la "Soft Computing", Actas del Simposio sobre Lógica Fuzzy y Soft Computing, (LFSC2005). Granada, 151-156 (2005).

5. J.L. Verdegay, , Ed. Fuzzy Sets-based Heuristics for Optimization. Studies in Fuzziness, Springer Verlag, (2003).

6. L.A. Zadeh, "Soft Computing and Fuzzy Logic", IEEE Software, 11(6), 48-56 (1994).

7. L.A. Zadeh, "Applied Soft Computing"”, Applied Soft Computing, 1, 1-2 (2001).

8. L.A. Zadeh, "Fuzzy Sets", Information and Control, 338-353 (1965).

9. E. Trillas, "Sobre funciones de negación en la teoría de conjuntos difusos", Stochastica, III-1, 47-59 (1979) (in Spanish). English version in Advances of Fuzzy Logic, S. Barro et al. (Eds.), Universidad de Santiago de Compostela, 31-43 (1998).

10. H. Bustince, M. Pagola, E. Barrenechea, J. Fernandez, P. Melo-Pinto, P. Couto, H.R. Tizhoosh, J. Monteroy, "Ignorance functions. An application to the calculation of the threshold in prostate ultrasound images", Fuzzy Sets and Systems, In Press, Cor- rected Proof, Available on line 5 April 2009, DOI: 10.1016/j.fss.2009.03.005.

11. H. Bustince, J. Montero, M. Pagola, E. Barrenechea, D.Gmez "A survey of interval-valued fuzzy sets", in: W.Pedrycz (Ed.), Handbook of Granular Computing,Wiley, New Jersey, (2008).

12. F. Herrera, E. Herrera-Viedma, "Aggregation operators for linguistic weighted information"”, IEEE Trans. on Systems, Man and Cybernetics, Part-A: Systems and Humans, 27, 646-656 (1997).

13. C.H. Wang, C.S. Cheng and T.T. Lee, "Dynamical optimal training for interval type-2 fuzzy neural network (T2FNN)", IEEE Transactions on Systems Man and Cybernetics: Part B, 34 (3), 1462-1477 (2004) .

14. R. Sambuc, "Function $\Phi$-Flous, Application a l'aide au Diagnostic en Pathologie Thyroidienne", These de Doctorat en Medicine, University of Marseille (1975).

15. K.U. Jahn, "Intervall-wertige Mengen", Mathematische Nachrichten 68, 115-132 (1975).

16. L.A. Zadeh, "The concept of a linguistic variable and its application to approximate reasoning - I", Information Sciences 8 199-249 (1975).

17. I. Grattan-Guinness, "Fuzzy membership mapped onto interval and many-valued quantities", Zeitschrift für mathematische Logik und Grundladen der Mathematik, 22, 149-160 (1976).

18. A. Dziech, M.B. Gorzalczany, "Decision making in signal transmission problems with interval-valued fuzzy sets", Fuzzy Sets and Systems 23(2) 191-203 (1987).

19. M.B. Gorzalczany, "A method of inference in approximate reasoning based on interval-valued fuzzy sets", Fuzzy Sets and Systems 21, 1-17 (1987).

20. M.B. Gorzalczany, "Interval-valued fuzzy controller based on verbal model of object"', Fuzzy Sets and Systems 28(1), 45-53 (1988).

21. M.B. Gorzalczany, "Interval-valued fuzzy inference involving uncertain (inconsistent) conditional propositions", Fuzzy Sets and Systems, 29(2), 235-240 (1989).

22. M.B. Gorzalczany, "An interval-valued fuzzy inference method. Some basic properties", Fuzzy Sets and Systems 31(2), 243-251 (1989).

23. I. B. Türksen, "Interval valued fuzzy sets based on normal forms", Fuzzy Sets and Systems 20(2), 191210 (1986).

24. I.B. Türksen, Z. Zhong, "An approximate analogical reasoning schema based on similarity measures and interval-valued fuzzy sets", Fuzzy Sets and Systems, 34, 323-346 (1990).

25. I.B. Türksen, D.D. Yao, "Representation of connectives in fuzzy reasoning: The view through normal forms", IEEE Trans. on Systems, Man and Cybernetics, 14, 191-210 (1984).

26. I.B. Türksen, "Interval-valued fuzzy sets and compen- 
satory AND”, Fuzzy Sets and Systems 51, 295-307 (1992).

27. I. B. Türksen, "Fuzzy normal forms," Fuzzy Sets and Systems 69, 319-346 (1995).

28. I. B. Türksen, "Interval-valued strict preference with Zadeh triples", Fuzzy Sets and Systems 78, 183-195 (1996).

29. G. Klir, B. Yuan, "Fuzzy Sets and Fuzzy Logic: Theory and Applications", Prentice Hall, Upper Saddle River, NJ, (1995).

30. J.M. Mendel, "Advances in type-2 fuzzy sets and systems", Information Sciences, 177, 84-110 (2007).

31. D. Dubois, Foreword, in: H. Bustince, F. Herrera, J. Montero (Eds.), Fuzzy Sets and Their Extensions: Representation, Aggregation and Models, Springer, New York, (2007).

32. H. Bustince, "Indicator of inclusion grade for intervalvalued fuzzy sets. Application to approximate reasoning based on interval-valued fuzzy sets", International Journal of Approximate Reasoning, 23(3) 137-209 (2000).

33. G. Deschrijver, E.E. Kerre, "On the composition of intuitionistic fuzzy relations", Fuzzy Sets and Systems, 136(3), 333-361 (2003).

34. Shyi-Ming Chen, Hui-Yu Wang, "Evaluating students answer scripts based on interval-valued fuzzy grade sheets", Expert Systems with Applications, 36(6), 9839-9846 (2009).

35. Dongrui Wu, J. M. Mendel, "A vector similarity measure for linguistic approximation: Interval type-2 and type-1 fuzzy sets", Information Sciences, 178(2), 381-402 (2008).

36. Sanz J, Fernandez A, Bustince H, F. Herrera, "A First Study on the Use of Interval-Valued Fuzzy Sets with Genetic Tuning for Classification with Imbalanced Data-Sets", 4th International Workshop on Hybrid Artificial Intelligence Systems, JUN 10-12, 2009 Salamanca, SPAIN Hybrid Artificial Intelligences Systems, Book Series: Lecture Notes in Artificial Intelligence, 5572, 581-588 (2009).

37. H. Hagras, "Type-2 FLCs: A new generation of fuzzy controllers", IEEE Computational Intelligence Magazine, 2, 30-43 (2007).

38. H. Hagras, "A hierarchical type-2 fuzzy logic control architecture for autonomous mobile robots", IEEE T. Fuzzy Systems, 12, 524-539 (2004).

39. H. K. Lamand, L. D. Seneviratne, "Stability analysis of interval type-2 fuzzy-model-based control systems", IEEE Transactions on Systems,Man and Cybernetics: Part B, 38(3), 617-628 (2008).

40. F. J. Lin, P. H. Chou, P. H. Shieh and S. Y. Chen, "Robust control of an LUSM-based XY-motion control stage using an adaptive interval type-2 fuzzy neural network", IEEE Transactions on Fuzzy Systems, 17(1), 24-38 (2009).
41. R. Sepulveda, O. Castillo, P. Melin, A. RodriguezDiaz, O. Montiel, "Experimental study of intelligent controllers under uncertainty using type- 1 and type-2 fuzzy logic", Information Sciences, 177, 2023-2048 (2007).

42. H. Bustince, E. Barrenechea, M. Pagola, J. Fernandez, "Interval-valued fuzzy sets constructed from matrices: Application to edge detection", Fuzzy Sets and Systems, 60(13), 1819-1840 (2009).

43. H.R. Tizhoosh, "Image thresholding using type-2 fuzzy sets", Pattern Recognition, 38, 2363-2372 (2005).

44. F. Liu, H. Geng, Y.-Q. Zhang, "Interactive fuzzy interval reasoning for smart web shopping", Applied Soft Computing, 5(4), 433-439.

45. T. Bilgi, "Interval-valued preference structures", $E u$ ropean Journal of Operational Research, 105162 183 (1998).

46. L. Martinez, J. Liu, Da Ruan, J-B. Yang, "Dealing with heterogeneous information in engineering evaluation processes", Information Sciences, 177, 15331542 (2007).

47. V. Khatibi, GA Montazer, "Intuitionistic fuzzy set vs. fuzzy set application in medical pattern recognition", Artificial Intelligence in Medicine, 47(1), 43-52 (2009).

48. M.E. Yuksel, M. Borlu, "Accurate Segmentation of Dermoscopic images by Image Thresholding based on Type-2 Fuzzy Logic", IEEE Transactions on Fuzzy Systems, 17(4), 976-982 (2009).

49. X. S. Xia and Q. L. Liang, "Crosslayer design for mobile ad hoc networks using interval type-2 fuzzy logic systems", International Journal of Uncertainty, Fuzziness and Knowledge-Based Systems, 16(3), 391-408 (2008).

50. Byung-In Choi, Frank Chung-Hoon Rhee, Interval type-2 fuzzy membership function generation methods for pattern recognition, Information Sciences, 179, (13), (2009), 2102-2122.

51. R. Martinez, O. Castillo, L.T. Aguilar, "Optimization of interval type- 2 fuzzy logic controllers for a perturbed autonomous wheeled mobile robot using genetic algorithms", Information Sciences, 179(13), 2158-2174 (2009).

52. L. A. Zadeh, "Fuzzy logic = computing with words", IEEE Trans. on Fuzzy Systems, 4, 103-111 (1996).

53. J.M. Mendel, R.I.J. Robert, "Type-2 fuzzy sets made simple", IEEE Transactions on Fuzzy Systems, 10(2), 117-127 (2002).

54. J.M. Mendel, H. Wu, "Type-2 Fuzzistics for Symmetric Interval Type-2 Fuzzy Sets: Part 1, Forwarrd Problems", IEEE Transactions on Fuzzy Systems, 14(6), 781-792 (2006).

55. J.M. Mendel, "Uncertain Rule-Based Fuzzy Logic Systems", Prentice-Hall, Upper Saddle River, NJ, 
(2001).

56. J. M. Mendel " Historical reflections on perceptual computing" , Proceedings of the 8th International FLINS Conference, FLINS'08, World Scientific, 181187, (2008). International Journal of Intelligent Systems, 13, 2-3, 287-300 (2008).

57. I.B. Türksen, "Meta-linguistic axioms as foundation for computing with words", Information Sciences, 177, 332-359 (2006).

58. I.B. Türksen, "Type-2 representation and reasoning for CWW", Fuzzy Sets and Systems, 127 17-36 (2002).

59. J. M. Mendel, "Computing with words: Zadeh, Tur- ing, Popper and Occam”, IEEE Computational Intelligence Magazine, 2, 10-17 (2007).

60. J.M. Mendel, "Computing with words and its relationships with fuzzistics", Information Sciences, 177, 988-1006 (2007).

61. G. Deschrijver, E.E. Kerre, "On the position of intuitionistic fuzzy set theory in the framework of theories modelling imprecision", Information Sciences, 177, 1860-1866 (2007).

62. J. Montero, D. Gómez, H. Bustince, "On the relevance of some families of fuzzy sets", Fuzzy Sets and Systems, 158(22), 2429-2442 (2007). 